\title{
Glycan-related gene expression signatures in human metastatic hepatocellular carcinoma cells
}

\author{
XIAONAN KANG ${ }^{1,2^{*}}$, NIAN WANG ${ }^{2 *}, \mathrm{CHEN} \mathrm{PEI}^{1}, \mathrm{LU} \mathrm{SUN}^{1}$, \\ RUIXIA SUN ${ }^{1}$, JIE CHEN ${ }^{1}$ and YINKUN LIU ${ }^{1}$ \\ ${ }^{1}$ Liver Cancer Institute of Zhongshan Hospital and Institute of Biomedical Science, Fudan University, Shanghai; \\ ${ }^{2}$ Department of Physiology, Chengdu Medical College, Sichuan, P.R. China
}

Received August 18, 2011; Accepted October 14, 2011

DOI: $10.3892 /$ etm.2011.430

\begin{abstract}
Human hepatocellular carcinoma (HCC) ranks second in cancer mortality in China; recurrence and metastasis have been the cause of the high mortality. Glycans on the cell surface play a pivotal role in tumor metastasis. The global alteration in the structure and composition of $\mathrm{N}$-glycans during HCC metastasis remains unknown. To understand glycan alterations of glycoproteins by correlating the glycosyltransferase expression profile with glycan structure, we systematically used glycan profiling tools: glycogene microarray analyses of 115 genes, including glycotransferases, glycosidases and nuclear sugar transporters and lectin chips to investigate the glycan-related gene expression signatures in the high metastatic potential HCC cell line, HCCLM3, in comparison to the HCC cell line, Hep3B, with low metastatic potential. Of the 115 genes, 18 genes were up-regulated in high metastatic potential HCCLM3 cells in comparison to Hep3B cells, while 11 genes were down-regulated. The differentially expressed genes, such as ST3GalI, FUT8, $\beta 3$ GalT5, MGAT3 and MGAT5, were mainly involved in the synthesis of N-glycan and glycolipids, particularly the sialyl Lewis antigen. The results of the glycogene microarray analysis were further validated by quantitative real-time PCR analysis and lectin-based analysis. The differentially expressed glycogenes identified in this study may provide new insights and represent novel factors for investigating the functional role of cell surface carbohydrate-mediated HCC metastasis.
\end{abstract}

Correspondence to: Dr YinKun Liu, Liver Cancer Institute of Zhongshan Hospital and Institute of Biomedical Science, Fudan University, 131 Dong An Road, Shanghai 200032, P.R. China E-mail: liu.yinkun@zs-hospital.sh.cn

*Contributed equally

Key words: glycan gene, metastasis, hepatocellular carcinoma, lectin array

\section{Introduction}

Cell membrane glycoproteins and glycolipids play a significant role in a number of biological functions such as cell-cell interactions, growth regulation, differentiation and malignant transformation. Metastasis is a selective process by which certain tumor cells disseminate to form secondary foci at distant sites $(1,2)$. Tumor cell metastasis requires alterations in membrane properties determined by cell surface glycoconjugates $(3,4)$. The biosynthesis of carbohydrate structures is tissue-specific and developmentally regulated by glycosyltransferases (5). Alterations in the expression and activity of glycosyltransferases could result in structural variations of glycans on glycoproteins in the cell membrane, which in turn alter their interaction with carbohydrate-binding proteins and modulate the role of the membrane glycan in cell adhesion and signal transduction events required for cell motility. Simultaneous analysis of the expression of all glycan-related genes clearly gives the advantage of enabling a comprehensive view of the genetic background of the glycobiological changes in cancer cells.

Hepatocellular carcinoma (HCC) is an aggressive cancer with poor prognosis, ranking fourth in the world and second in China in cancer mortality, despite the advances in surgery. High recurrence and metastasis rates have been the reason for the poor long-term survival (6). However, the universal alteration in the structure and composition of glycans in the cell membrane protein structure has not yet been clearly defined in HCC metastasis.

The goal of this study was to identify differentially expressed glycosyltransferases in HCC metastasis-specific glycogenes and glycan structures using DNA microarray analysis and lectin chips. A significant advantage of gene expression analysis is that it constitutes a simple and uniform process for material handling. To date, the expression profile of glycosyltransferases during metastasis has not been determined. In the present study, using a glycogene microarray approach that detects the transcript levels of enzymes regulating glycosylation between the hepatoma cell lines, HCCLM3 (high metastasis) and Hep3B (low metastasis), while the glycan profile signature of the membrane protein labeled with $\mathrm{Cy} 3$ was assayed through the lectin-based array. Through ingenuity pathways analysis (IPA), differentially expressed glycosyltransferases mainly 
played a role in biological processes such as angiogenesis, cell adhesion and invasion. These changes were possibly related to the metastatic potential of HCCLM3 cells.

\section{Materials and methods}

Materials. RPMI-1640, fetal bovine serum, glutamine, guaninium isothiocyanate/phenol/chloroform and ethidium bromide were purchased from Gibco BRL (Auckland, NJ, USA); The ProteoExtract ${ }^{\mathrm{TM}}$ native membrane extraction kit was from the Calbiochem company of Merck KGaA (Darmstadt, Germany). The Cy3-monofunctional dye pack was from GE Healthcare (Piscataway, NJ, USA). The 2D-quantification kit was from GE Healthcare.

Cell culture. The low-metastatic HCC cell line, Hep3B, a gift from Cornell University, was cultured with $\alpha$-MEM medium (Gibco BRL). The high-metastatic HCC cell line, HCCLM3, established in our institute, was cultured with Dulbecco's modified Eagle's medium (Gibco BRL). All culture media were supplemented with $10 \%$ fetal bovine serum (HyClone, Logan, UT, USA) and placed in an incubator containing $5 \% \mathrm{CO}_{2}$ at $37^{\circ} \mathrm{C}$.

Glycogene microarray fabrication. The glycogene microarray is an oligonucleotide microarray designed by the CapitalBio company (Beijing, China). The array contains 115 genes including 90 glycosyltransferases, 20 glycosidases and 5 nuclear sugar transporters. Individual 70-mer oligonucleotides complementary to sequences within these human mRNAs were designed and prioritized using stringent selection criteria, including minimal secondary structure, minimal homology to other genes in the available human genomic databases, no low complexity or repeat regions. The individual oligonucleotide probe was printed 5 times on chemical modification glass slides and $15 \times 15$ spot configuration of each subarray. The spot diameter was $120 \mu \mathrm{m}$, and the distance from center to center was $280 \mu \mathrm{m}$.

RNA preparation and chip hybridization. Total RNA from the cells was extracted with TRIzol reagent (Invitrogen, Carlsbad, CA, USA), and purified with the NucleoSpin RNA clean-up kit (Macherey-Nagel, Germany). Total RNA (40 $\mu \mathrm{g})$ was labeled by reverse transcription using Superscript II transcriptase, oligo(dT) primer, Cy3dCTP and Cy5dCTP for Hep3B and HCCLM3, respectively. The labeled cDNAs were then hybridized to microarrays at $42^{\circ} \mathrm{C}$ for $12-18 \mathrm{~h}$. The microarrays were scanned using a dual laser scanner (LuxScan 10K/A; CapitalBio). The figure signal was then transformed to a digital signal using image analysis software (LuxScan3.0; CapitalBio). Signal intensities for each spot were calculated by subtracting the local background from the total intensities. The experimental data were analyzed with the QuantArray software package. All the procedures were performed according to the manufacturer's instructions.

Data acquisition and statistical analysis. Arrays were scanned at $5 \mu \mathrm{m}$ resolution on a LuxScan 10KA double channel scanner (CapitalBio) at the maximal laser power that produced no saturated spots. The adaptive threshold method was used to differentiate the spot from the background and the spot intensity was determined using median pixel intensity. Prior to normalization, quality confidence measurements (spot diameter, spot area, array footprint, spot circularity, signal:noise ratio, spot uniformity, background uniformity and replicate uniformity) were calculated for each scanned array and spots were flagged that did not pass stringent selection criteria.

Each sample was labeled by $\mathrm{Cy} 3$ and $\mathrm{Cy} 5$, for each probe there were 5 repeats in each array. Transformed expression values for each probe were averaged to get a single expression value. The statistically significant changes in gene expression were then identified using the permutation-based, significance analysis of microarrays (SAM) algorithm (v2.20; Stanford University, CA, USA), which reports the median false discovery rate (FDR) as the percentage of genes in the identified gene list that are falsely reported as showing statistically significant differential expression. The cut-off value for significance in these experiments was set at a 5\% FDR at a specified 2-fold change.

Quantitative real-time PCR analysis. The expression levels of selected genes were validated and analyzed by real-time PCR using Brilliant SYBR-Green qRT-PCR Master Mix (Stratagene, Santa Clara, CA, USA) on a BioRad Real-Time PCR System. All primer sets were designed across intron:exon boundaries (Table I), with individual primer concentrations and final amplification conditions optimized for each gene. Dissociation curves were performed on all reactions to assure product purity. Original input RNA amounts were calculated by comparison to standard curves using a purified PCR product as a template for the mRNAs of interest and were normalized to the amount of actin. Experiments were performed in triplicate for each data point.

Membrane protein labeling and extraction. A total of 5-10x $10^{6}$ cells were incubated with $0.5 \mathrm{M}$ EDTA in phosphatebuffered saline (PBS) (pH 7.2) for $10 \mathrm{~min}$, and collected by centrifugation at $500 \mathrm{xg}$ at $4^{\circ} \mathrm{C}$ for $10 \mathrm{~min}$. The cells were then washed with PBS twice to remove EDTA, and resuspended in $200 \mu 1$ PBS.

A vial of Cy3-monofunctional dye powder (GE Healthcare) was dissolved with $50 \mu \mathrm{l}$ DMSO. Dye/DMSO mixture (10 $\mu \mathrm{l})$ was added to $200 \mu \mathrm{l}$ cell suspension, and incubated in the dark at $4^{\circ} \mathrm{C}$ for $2 \mathrm{~h}$ to label the intact cell membrane. The labeled cells were washed with PBS 3 times and centrifuged at $600 \mathrm{x} \mathrm{g} 4^{\circ} \mathrm{C}$ for $10 \mathrm{~min}$ to remove redundant fluorescent dyes. Then the labeled samples were quickly frozen in liquid nitrogen and stored at $-80^{\circ} \mathrm{C}$ in the dark. The ProteoExtract ${ }^{\mathrm{TM}}$ native membrane extraction kit (Calbiochem) was adopted to extract cell membrane proteins according to the manufacturer's instructions. 2D-quantification kit (GE Healthcare) was used to determine the protein concentration.

Lectin array analysis. To detect the glycan alteration of the membrane protein, Cy3-labeled membrane glycoprotein was incubated with lectin array. A total of 13 types of tumor-associated lectins (Vector Laboratories, Burlingame, CA, USA) were dissolved with chip-spotting buffer (CapitalBio) at a concentration of $1 \mathrm{mg} / \mathrm{ml}$, and spotted on a gel-substrate chip using the microarray printing robot, Smart Arrayer- 48 (CapitalBio). The diameter of each point was $150 \mu \mathrm{m}$, and 
Table I. The primers of validated glycogenes.

\begin{tabular}{llc}
\hline Gene & \multicolumn{1}{c}{$\begin{array}{c}\text { Forward }\left(5^{\prime} \rightarrow 3^{\prime}\right) \\
\text { reverse }\left(5^{\prime} \rightarrow 3^{\prime}\right)\end{array}$} & $\begin{array}{c}\text { Product } \\
(\mathrm{bp})\end{array}$ \\
\hline MGAT5 & $\begin{array}{l}\text { GCTGCCCAACTGTAGGAGAC } \\
\text { GAATCAAGGACTCGGAGCAT }\end{array}$ & 127 \\
\multirow{2}{*}{ MGAT3 } & GCCTCACCTTGGGAGTTATC & 286 \\
& GCATCATTGGGTAGCGTCTG & \\
FUT8 & AGCGAACACTCATCTTGGAA & 257 \\
& TTGACAAACTGAGACACCCA & \\
ST3GalI & CACGAATGGCGTTGGTCTAC & 131 \\
& CTCAATCAAAAGGGATGGCA & \\
33GalT5 & CAGATAACCCGTGGGGATAG & 133 \\
& GCACCAAGTGGGAACTAATC & \\
$\beta$-actin & CATGTACGTTGCTATCCAGGC & 250 \\
& CTCCTTAATGTCACGCACGAT & \\
\hline
\end{tabular}

Fold change expressed following normalization to the housekeeping gene, $\beta$-actin.

the distance between two points was $400 \mu \mathrm{m}$. Each lectin point had 4 repeats. The chip was then incubated in a vacuum chamber with humidity greater than $80 \%$ at $25^{\circ} \mathrm{C}$ overnight to immobilize the lectins.

To block the non-specific binding sites on the chip, $100 \mu 1$ of $0.1 \%$ Tris- $\mathrm{HCl}$ were added $\left(25^{\circ} \mathrm{C}, 1 \mathrm{~h}\right)$ and then washed with $0.1 \%$ TBS-Tween-20 solution ( $5 \mathrm{~min}, 3$ times). Next, $10 \mu \mathrm{g}$ of Cy3-labeled glycoprotein suspended in TBS (1 mM $\mathrm{Ca}^{2+}, 2 \mathrm{mM} \mathrm{Mg}{ }^{2+}$ ) were added to each chip in a final volume of $40 \mu \mathrm{l}$ and incubated at $25^{\circ} \mathrm{C}$ for $2 \mathrm{~h}$ with gentle rocking. The unbound glycoprotein was washed off with cold $0.1 \%$ TBS-Tween-20 solution (15 min, 3 times) and immersed in PBS for $5 \mathrm{~min}$. The chip was then centrifuged at 1,500 rpm to get rid of residual liquid and prepare it for scanning.

The binding fluorescence signals of the glycoproteins with lectins on the gel slide were obtained with the fluorescence scanner LuxScan 3.0 (CapitalBio). The net intensity value for each spot was calculated by subtracting the background value. Median rectification was used to calculate the dye-bias-corrected ratios. Median corrected data were used to calculate the q-value by T-statistic analysis with SAM version 2.10 software.

Fluoresceinated lectin staining. The HCC cells lines Hep3B and HCCLM3 were cultured in 24-well plates (Gibco, BRL), and wells at $60-80 \%$ confluence were selected. First, the cells were fixed with ice-cold paraformaldehyde for $15 \mathrm{~min}$ and rinsed three times with $\mathrm{PBS}$, then the cells were incubated with biotinylated LCA, PHA-E and RCA-1 (5 $\mu \mathrm{g} / \mathrm{ml}$ in PBS-T with 5\% BSA) for $30 \mathrm{~min}$. After rinsing 3 times with PBS, cells were then incubated with Streptavidin-Alexa fluor 488 (1:1,000 dilutions in PBS-T with 5\% BSA; Molecular Probes, Leiden, Holland) for $30 \mathrm{~min}$ and rinsed 3 times with PBS. Finally, the cells were incubated with the DAPI (300 nM

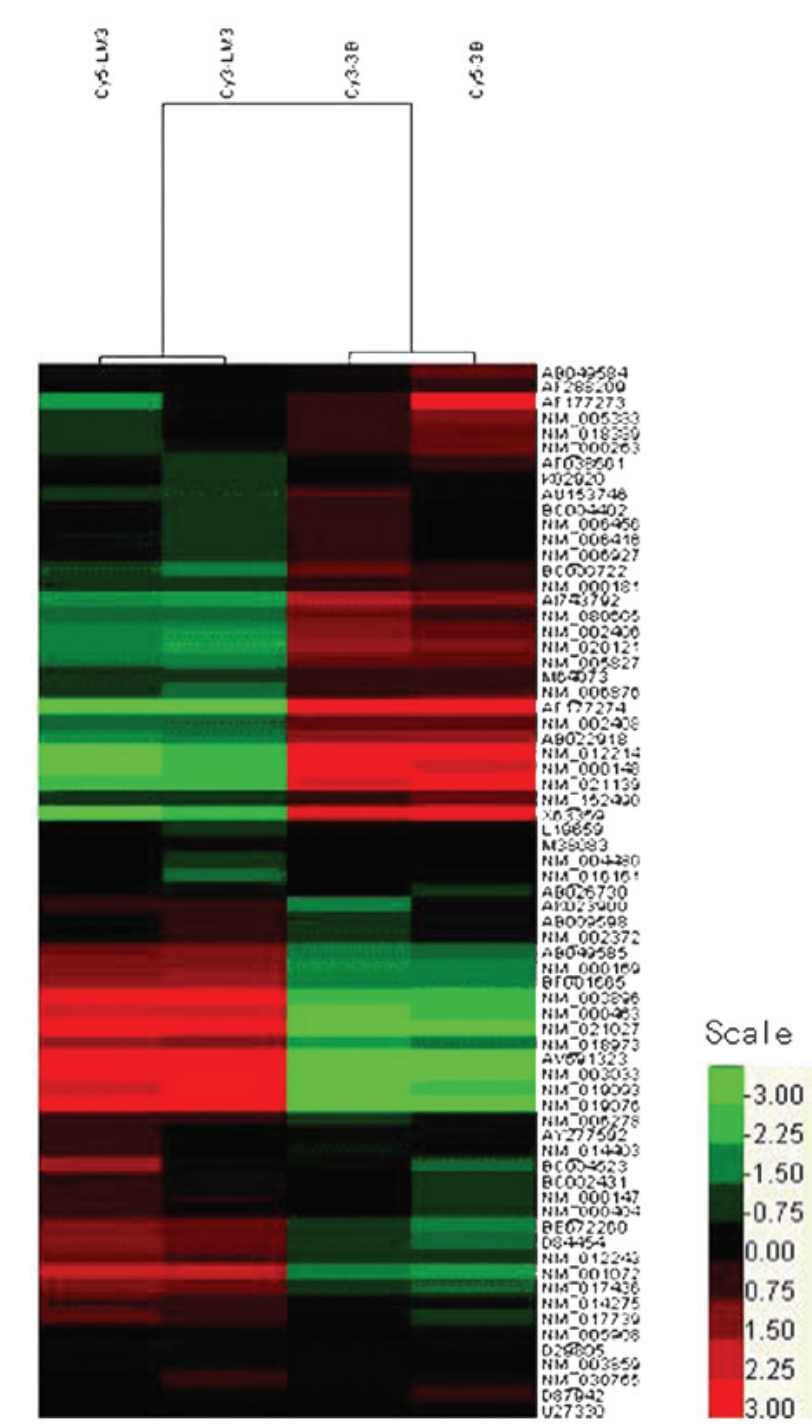

Figure 1. Hierarchical clustering of glycan gene expression. Hep3B and HCCLM3 cells are well-separated, indicating that glycan genes are differentially expressed between the two cell lines.

in PBS; Molecular Probes) for $5 \mathrm{~min}$ and washed with PBS 3 times. The images for all the cell lines were then captured using an inverted microscope (IX81; Olympus, Tokyo, Japan) under the same conditions.

Functional study of differentially expressed genes by IPA. Differentially expressed genes were uploaded to the IPA database to explore the possible biological function in HCC metastasis. The IPA tool is capable of mapping a gene network based on the information stored in the IPA database or PubMed. With this network, the correlation between various genes and the biological function of target genes in a certain disease or biological process is capable of being elucidated.

\section{Results}

Identification of differentially expressed glycosyltransferase and glycosidase genes. The differentially expressed genes were visualized as a heat map using Cluster and TreeView software (Fig. 1). This removes all genes that have missing values 


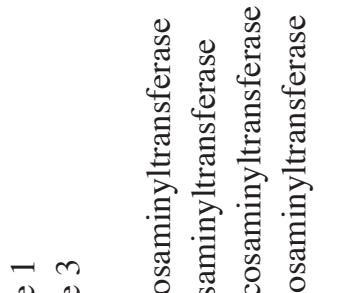

:

产

究䒔

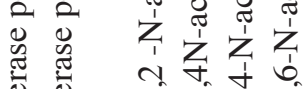

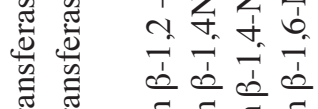

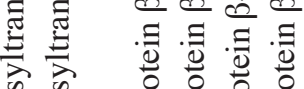

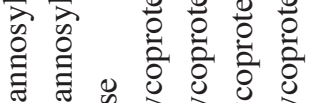

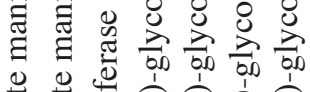

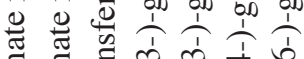

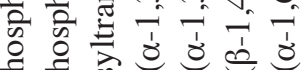

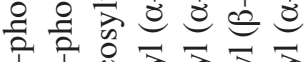

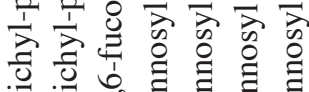

它 它

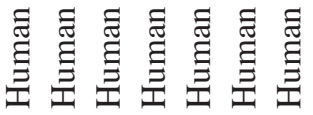

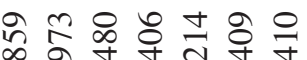

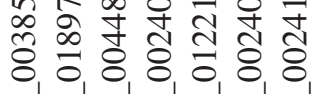

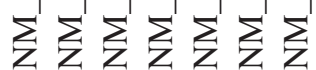

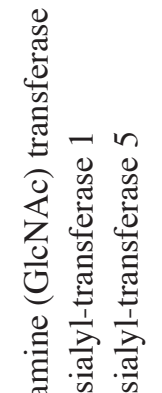

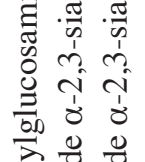

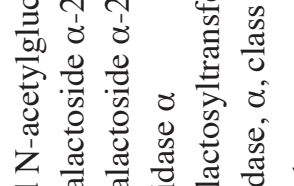

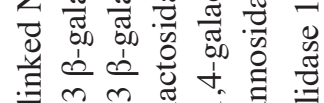

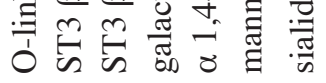

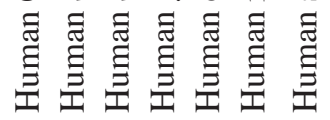

ำ

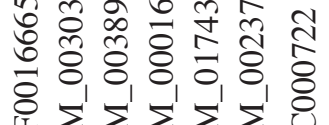

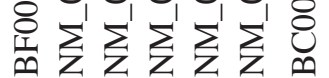

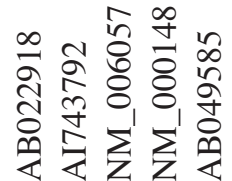

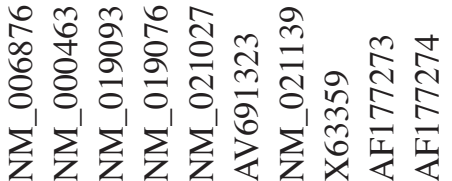

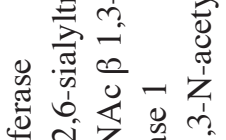

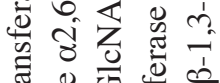

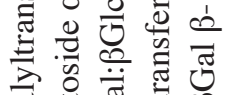

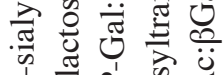

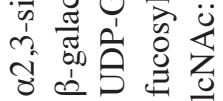

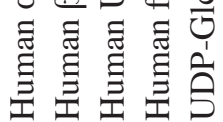

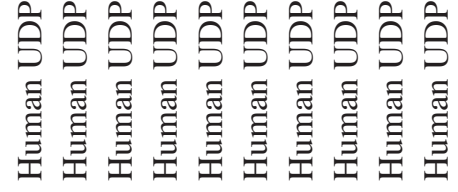

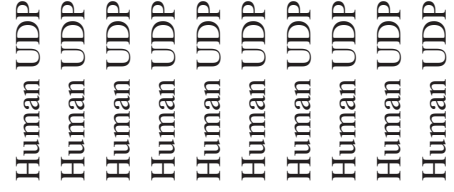

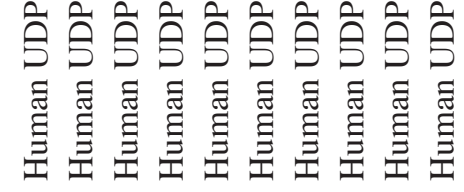

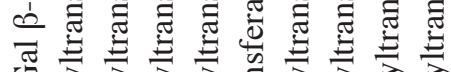

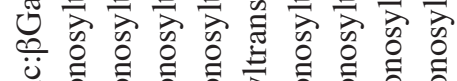

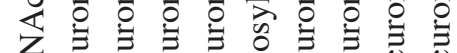

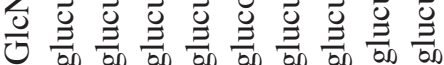

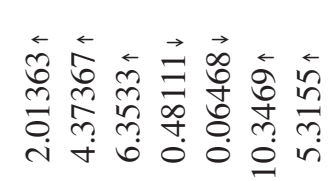

$\overleftarrow{m} \overleftarrow{n} \overleftarrow{n} \overleftarrow{n} \overleftarrow{\sigma} \overleftarrow{\sigma} \vec{\sigma}$

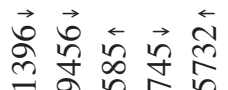

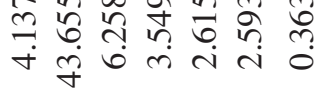

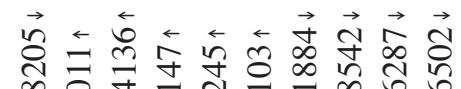

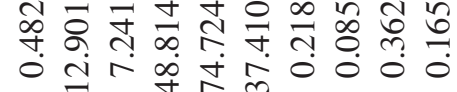

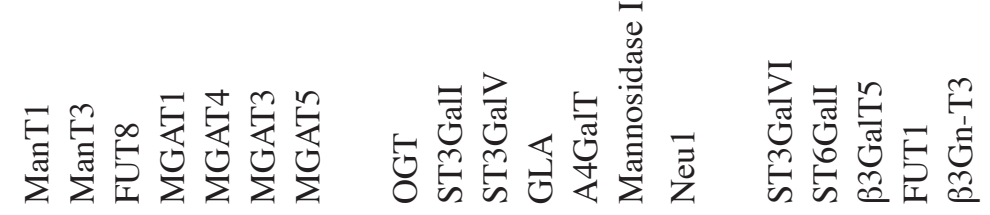

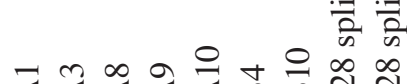
$\frac{8}{0}: \frac{0}{2}$

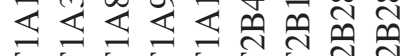

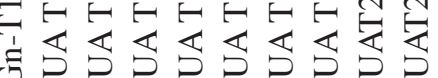

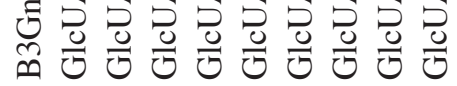




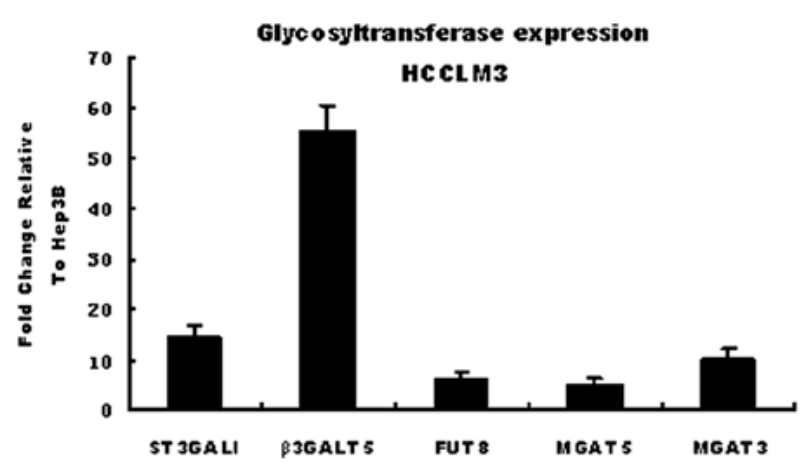

Figure 2. Relative mRNA levels of glycosyltransferase gene expression in HCCLM3 and Hep3B cells using real time-PCR.

in more than $20 \%$ of the columns. The color-based view also demonstrates that HCCLM3 and Hep3B cells present distinct gene expression profiles from each other. Using the difference $\leq 0.5$ or $\geq 2$ as a criteria, a total of 29 genes were found to be differentially expressed between Hep3B and HCCLM3 cells. A total of 18 genes were found to be up-regulated, while 11 genes were down-regulated. Table II demonstrates the 18 up-regulated genes and 11 down-regulated genes, which have been grouped in the synthesis of N-glycan, O-glycan, glycosaminoglycans and hyaluronan synthase using the Kyoto Encyclopedia of Genes and Genomes (KEGG) database according to the respective function in glycan synthesis.

qRT-PCR validation of differentially expressed genes in cell lines. A total of 5 glycosyltransferases including ST3GalI, FUT8, MGAT3, MGAT5 and $\beta 3$ GalT5 were selected to verify the results of the GT oligonucleotide array by methods of realtime PCR. $\beta$-actin was used as a reference. These genes were selected mainly due to their correlation with lectins MAL-I, MAL-II, LCA and PHA-E. The affinity of the HCCLM3 and Hep3B cell lines for these lectins differs. The results of real-time PCR proved that the expressions of ST3GalI, FUT8, MGAT3, MGAT5 and $\beta 3$ GalT5 were up-regulated to a different extent in HCCLM3, supporting the data from the chip array (Fig. 2).

Alteration in transcript levels of glycosyltransferases is associated with corresponding changes in the glycan structures of glycoproteins. To examine whether the differential glycosyltransferases have led to the alteration of the related glycan structure, the lectin array and fluorescence labeling lectin staining were selected to compare the cell surface glycan profile between Hep3B and HCCLM3 cells. It was found that the cell line with a high metastatic tendency exhibited a higher affinity for LCA, MAL-I, MAL-II, WGA and PHA-E, and a lower affinity for RCA-I (Fig. 3A). The core fucose structure, sialic acid structure (mainly in the 2,3 link), $\mathrm{N}$-acetylglycosamine and bisecting oligo-glycan were up-regulated, while end- $\beta 1$ 4-linked galactose was down-regulated (Fig. 3B). Cell surface lectin affinity chemistry using biotin-labeled LCA, PHA-E and RCA-I reconfirmed the result of the lectin array (Fig. 3C).

Combined analysis of the results obtained from the lectin affinity profile and glycogene microarray, showed the up-regulation of FUT8, MGAT3 and ST3GalI in HCCLM3 cells matched with the structure of corefucosylation, bisecting $\mathrm{N}$-acetylglucosamine and $\alpha 2-3$ sialic acid. Therefore, the affinity increased in the LCA, PHA-E, MAL-I, MAL-II and WGA affinity assay.

Functional sub-network of differential expressed genes by IPA. The Search Tool for the Retrieval of Interacting Proteins (STRING) database was used to establish the gene function network. It was found that certain genes were responsible for a change of glycosylation and expression of adhesion molecules or their receptors such as MGAT3, to influence cell adhesion. Genes including ST3Gals, $\beta 3$ GalT5, $\beta 3 \mathrm{Gn}$-Ts and FUT8 were involved in sialyl Lewis antigen synthesis. Genes such as MGAT5 may play a role in the blood vessel growth factor secretion to promote angiogenesis as well as to regulate the expression of TGF- $\beta$, and affect a variety of signaling pathways such as apoptosis (Fig. 4).

\section{Discussion}

Our aim in this study was to identify differentially expressed glycosyltransferases between two cell lines with different metastatic potential, and to validate whether the glycan alteration in membrane proteins between the 2 cell lines was related to the glycogene changes. Although there are various technologies that are capable of decoding glycan structure, such as mass spectrometry, affinity chromatography technology, nuclear magnetic resonance and carbohydrate microarray, none of them have enabled systematic analysis of the altered carbohydrate structure. The microarray strategies, including lectin, glycan and glycogene arrays, represent a set of essential tools to speed up the development of functional glycomics (7). Exploring gene expression is experimentally more tractable than comprehensively characterizing glycan structures associated with metastatic tendency. The expression alterations in glycogenes have previously been examined using focused microarrays or PCR arrays designed to specifically measure the relevant transcripts $(7,8)$. In this study, we demonstrate the use of glycan gene expression profiling and membrane glycoprotein lectin array assay to capture the metastasis-related information of HCC. The gel-substrate lectin microarray was established in our laboratory, by which the altered glycosylation was capable of being analyzed in a rapid, sensitive and high-throughput manner. In a previous study, we screened the glycan profiles of a number of glycoprotein standards and compared the glycan profiles of AFP derived from various sources (9).

Through the profile comparison we found a group of differences in glycogenes between the 2 HCC cell lines, which were further categorized according to their specific function listed in Table II. The alteration mainly focused on the aspects of $\mathrm{N}$-glycan, O-glycan, sialic acid change, Lewis structure and glycosaminoglycans. Of these 18 genes, genes related to N-glycan synthesis are: MGAT1, MGAT3, MGAT4 and MGAT5 mainly participate in branching of N-glycan, FUT8 is responsible for core fucosylation formation through an $\alpha 1,6$ link, and ManT1 and ManT3 provide dolichol phosphate mannose involving the N-glycan precursor. Branched N-glycans, such as bisecting GlcNAc, $\beta 1,6$ GlcNAc and core fucose ( $\alpha 1,6$ fucose), are enzymatic products 
A Hep3B

HCCLM3

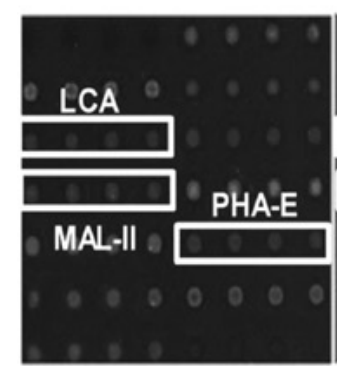

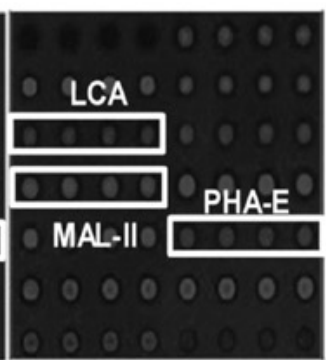

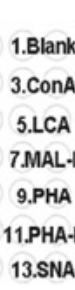

C

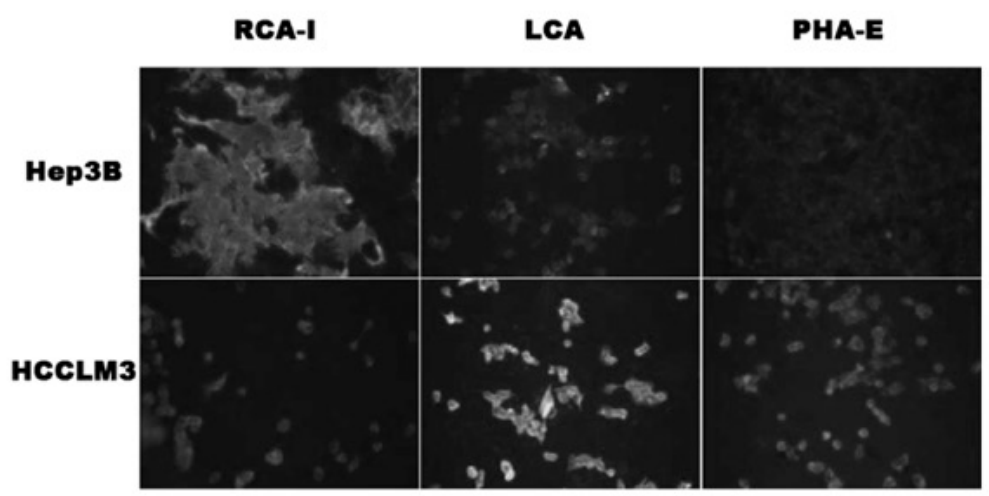

Figure 3. Differential membrane glycoprofiling of Hep3B and HCCLM3 cells. (A) Cy3-labeled membrane proteins of Hep3B and HCCLM3 cells were allowed to bind with lectin microarray. Bound membrane proteins were scanned with a fluorescent scanner. (B) Differential glycan profiling of Hep3B and HCCLM3 cells from lectin array. Predicted representative differential structures expressed in Hep3B and HCCLM3 were shown by monosaccharide symbols. "P<0.01. (C) Validation of microarray results of Hep3B and HCCLM3 cells by means of fluorescence labeling lectin staining.

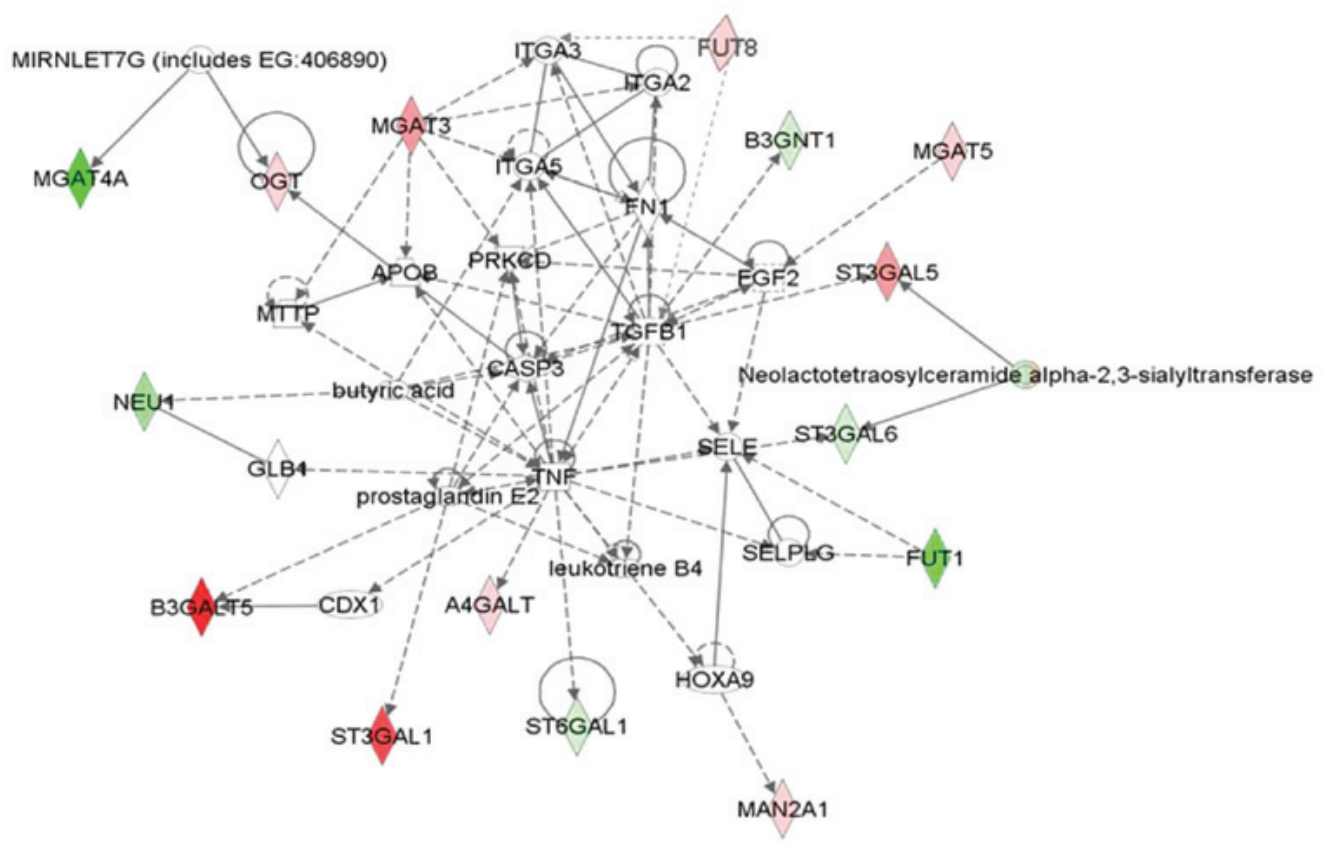

Figure 4. Functional subnetwork of differentially expressed glycogenes. The networks of genes with altered expression in HCCLM3 cells compared to Hep3B. Differentially expressed genes listed in Table II were analyzed by the IPA tool. The network shown was found to be significantly associated with cell adherence, apoptosis and angiogenesis. Gene products belonging to different functional classes are displayed with various shapes. Colored nodes are derived from the glycan gene array, and the white nodes are from the IPA database. The green represents a decreased expression in HCCLM3 whereas red indicates an increased expression. Solid lines represent direct interactions between 2 nodes and dashed lines represent indirect interactions. Lines beginning and ending on the same node mean self-regulation. Arrowheads mean node A acts on node B, while the edge without arrowheads means a binding relationship between two nodes.

of N-acetylglucosaminyltransferase III (encoded by MGAT3), $\mathrm{N}$-acetylglucosaminyltransferase V (encoded by MGAT5) and $\alpha-1,6$ fucosyltransferase (encoded by FUT8), respectively. These structures are highly associated with various biological func- 
tions of cell adhesion molecules, including cell adhesion and cancer metastasis (10). These structures on proteins, including growth factor receptors and integrins, are shown to enhance growth signaling in motile tumor cells. Core fucosylation is of special interest due to its ability to modulate growth and development through altering functional properties of integrins. For example it has been shown that core decoration by $\alpha 1,6$ fucose of certain motifs of the $\alpha 3 \beta 1$ integrin complex is essential for its association and activity (11). Bisecting GlcNAc structure synthesized by MGAT3 was increased in HCCLM3 cells, data from the lectin microarray and GTs chip supported this finding. The bisecting GlcNAc structure has been reported to be correlated with the onset of hepatoma in rats and humans, and also a characteristic structure of AFP derived from liver cancer $(12,13)$. The overexpressed bisecting GlcNAc structure on integrin down-regulated the binding capacity of integrin with its ligand, leading to reduced cell adhesion and cell migration (14). The introduction of the bisecting GlcNAc structure into the CD44 adhesion molecule promoted tumor formation and metastasis (11).

In the current study, we found the increased expression of ST3GalI and ST3GalV in HCCLM3 cells compared to Hep3B cells, which synthesizes the $\alpha 2-3$-linked sialic acid. The result from the increased lectin affinity fluorescent intensity of MAL-I, MAL-II and WGA for HCCLM3 also shows this alteration. According to previous reports, the increased sialic acid reduces the adhesion of tumor cells to the cell matrix, facilitating the shedding of tumor cells from the primary sites into the blood. $\alpha 2-3$-linked sialic acid plays a role in the composition of the sialyl Lewis structure $(15,16)$. Once tumor cells release into the blood, they bind to interleukin-secreting cells and platelets through the interaction of the sialyl Lewis structure on the tumor cell surface with the selectins on interleukin-secreting cells and platelets, leading to hematogenous metastasis. Tumor cells may also be settled in a new place through the binding of the sialyl Lewis structure with the selectin expressed on endothelial cells (17). Therefore, ST3GalI and ST3Galv may promote HCC cell metastasis by regulating cell adhesion through the $\alpha 2-3$-linked sialic acid structure.

Another finding is that UDP glucuronosyltransferase family members (UGTs) differ in the two liver cancer cell lines. The UGTs are a family of enzymes, the substrates of which include drugs, xenobiotics and products of endogenous catabolism. The main source of the majority of UGT enzymes is the liver, a major organ in the detoxification and inactivation of compounds. Due to the importance of UGTs in forming conjugates between steroids and glucuronic acid, thereby inactivating them and targeting them for removal, the estrogen-induced up-regulation of UGTs might affect estrogen and androgen concentrations, thereby reducing their signaling in breast cancer cells (18). Hepatic UGT mRNA levels are reduced while the tissue is inflamed, but they are not affected in the non-inflamed, chronically diseased liver (19). To date, there has been no report on the correlation between liver cancer and UGTs. However, UGT mRNA and protein levels are down-regulated or are completely absent in a number of cancer tissues such as those in the breast and prostate cancer (20).

Through protein interaction analysis, we observed two significant nodes in the IPA map: transforming growth factor- $\beta$ (TGF- $\beta$ ) and tumor necrosis factor- $\alpha$ (TNF- $\alpha)$. The function of TGF- $\beta$ is affected by glycosyltransferase FUT8 and MGAT5 (21), and it in turn has an impact on the function of other nodes such as TNF- $\alpha$. TGF- $\beta$ and TNF- $\alpha$ are key molecules involved in numerous biological functions, such as cell proliferation, differentiation and apoptosis. The involvement of TGF- $\beta$ and TNF- $\alpha$ in the glycogene IPA map indicates that glycogene tumor metastasis is regulated by a variety of mechanisms in collaboration, and those involved factors are mutually affected.

It should be noted that this study has examined only the cell lines with different metastatic potential and the clinical samples with different metastasis were not available for examination of the differential glycogenes. We could then collect the relative clinical samples to compare and validate the prognostic value of glycogenes.

The results of these analyses reveal that mRNA levels for a number of these genes differ significantly between the metastatic and non-metastatic HCC cell lines, indicating that synthesis, degradation and adhesion mediated by glycans may be altered drastically in HCC. Meanwhile we used lectin chips to validate the differences of the glycome. It was shown that increased expression of $\alpha 2-3$ sialic acid, core fucose and bisecting GlcNAc may be characteristic changes correlated with HCC metastasis. They may affect HCC metastasis by regulating cell adhesion, cell migration, angiogenesis and apoptosis. In the future, efforts should be made to understand how changes in mRNA levels of glycan genes affect a cell's glycome. The precise role of such altered glycan structures in disease remains to be elucidated.

\section{Acknowledgements}

We thank Mr. Yang Zhang (Institute of Biomedical Science, Fudan University) for his help in the bioinformatics analysis. We also thank Mr. HongMing Zhang and HouLong Cheng (CapitalBio, Beijing, China) for their technical help in the preparation of the lectin microarray and analysis of the results. This study was financially supported by the Major State Basic Research Development Program of China (973 Program) (2011CB910604), Natural Science Foundation of China (81101636), and the Ph.D. Programs Foundation of University Education of China (200802461155).

\section{References}

1. Chaffer CL and Weinberg RA: A perspective on cancer cell metastasis. Science 331: 1559-1564, 2011.

2. Hakomori S: Aberrant glycosylation in tumors and tumor-associated carbohydrate antigens. Adv Cancer Res 52: 257-331, 1989.

3. Ohtsubo K and Marth JD: Glycosylation in cellular mechanisms of health and disease. Cell 126: 855-867, 2006.

4. Wang FL, Cui SX, Sun LP, et al: High expression of alpha 2 , 3-linked sialic acid residues is associated with the metastatic potential of human gastric cancer. Cancer Detect Prev 32: 437-443, 2009.

5. Lauc G and Zoldos V: Protein glycosylation - an evolutionary crossroad between genes and environment. Mol Biosyst 6: 2373-2379,

6. Farazi PA and DePinho RA: Hepatocellular carcinoma pathogenesis: from genes to environment. Nat Rev Cancer 6: 674-687, 2006.

7. Comelli EM, Head SR, Gilmartin T, et al: A focused microarray approach to functional glycomics: transcriptional regulation of the glycome. Glycobiology 16: 117-131, 2006.

8. Nairn AV, York WS, Harris K, Hall EM, Pierce JM and Moremen KW: Regulation of glycan structures in animal tissues: transcript profiling of glycan-related genes. J Biol Chem 283: 17298-17313, 2008. 
9. Chen P, Liu Y, Kang X, Sun L, Yang P and Tang Z: Identification of $\mathrm{N}$-glycan of alpha-fetoprotein by lectin affinity microarray. J Cancer Res Clin Oncol 134: 851-860, 2008.

10. Saravanan C, Cao Z, Head SR and Panjwani N: Analysis of differential expression of glycosyltransferases in healing corneas by glycogene microarrays. Glycobiology 20: 13-23, 2010.

11. Zhao Y, Itoh S, Wang X, et al: Deletion of core fucosylation on alpha3beta1 integrin down-regulates its functions. J Biol Chem 281: 38343-38350, 2006.

12. Ohno M, Nishikawa A, Koketsu M, et al: Enzymatic basis of sugar structures of alpha-fetoprotein in hepatoma and hepatoblastoma cell lines: correlation with activities of alpha 1-6 fucosyltransferase and $\mathrm{N}$-acetylglucosaminyltransferases III and V. Int J Cancer 51: 315-317, 1992.

13. Sultan AS, Miyoshi E, Ihara Y, Nishikawa A, Tsukada Y and Taniguchi N: Bisecting GlcNAc structures act as negative sorting signals for cell surface glycoproteins in forskolin-treated rat hepatoma cells. J Biol Chem 272: 2866-2872, 1997.

14. Zhao Y, Sato Y, Isaji T, et al: Branched N-glycans regulate the biological functions of integrins and cadherins. FEBS J 275 1939-1948, 2008.

15. Dennis J, Waller C, Timpl R and Schirrmacher V: Surface sialic acid reduces attachment of metastatic tumour cells to collagen type IV and fibronectin. Nature 300: 274-276, 1982.
16. Ugorski M and Laskowska A: Sialyl Lewis(a): a tumor-associated carbohydrate antigen involved in adhesion and metastatic potential of cancer cells. Acta Biochim Pol 49: 303-311, 2002.

17. Fuster MM, Brown JR, Wang L and Esko JD: A disaccharide precursor of sialyl Lewis X inhibits metastatic potential of tumor cells. Cancer Res 63: 2775-2781, 2003.

18. Harrington WR, Sengupta S and Katzenellenbogen BS: Estrogen regulation of the glucuronidation enzyme UGT2B15 in estrogen receptor-positive breast cancer cells. Endocrinology 147: 3843-3850, 2006.

19. Congiu M, Mashford ML, Slavin JL and Desmond PV: UDP glucuronosyltransferase mRNA levels in human liver disease. Drug Metab Dispos 30: 129-134, 2002.

20. Zhang ZM, Yang XY, Yuan JH, Sun ZY and Li YQ: Modulation of NRF2 and UGT1A expression by epigallocatechin-3-gallate in colon cancer cells and BALB/c mice. Chin Med J (Engl) 122: 1660-1665, 2009.

21. Herr P, Korniychuk G, Yamamoto Y, Grubisic K and Oelgeschlager M: Regulation of TGF-(beta) signalling by $\mathrm{N}$-acetylgalactosaminyltransferase-like 1 . Development 135 : 1813-1822, 2008. 